\title{
Stochastic Chaos in a Turbulent Swirling Flow
}

\author{
D. Faranda \\ LSCE-IPSL, CEA Saclay l'Orme des Merisiers, CNRS UMR 8212 CEA-CNRS-UVSQ, \\ Université Paris-Saclay, 91191 Gif-sur-Yvette, France
}

\author{
Y. Sato* \\ Research Institute for Electronic Science/Department of Mathematics, Hokkaido University, \\ N20 W10, Kita-ku, Sapporo, Hokkaido 001-0020, Japan
}

\author{
B. Saint-Michel \\ Univ Lyon, Ens de Lyon, Univ Claude Bernard, CNRS, Laboratoire de Physique, F-69342 Lyon, France
}

C. Wiertel, V. Padilla, B. Dubrulle, and F. Daviaud ${ }^{\dagger}$

SPEC, CEA, CNRS, Université Paris-Saclay, CEA Saclay 91191 Gif sur Yvette cedex, France

(Received 19 July 2016; published 7 July 2017)

\begin{abstract}
We report the experimental evidence of the existence of a random attractor in a fully developed turbulent swirling flow. By defining a global observable which tracks the asymmetry in the flux of angular momentum imparted to the flow, we can first reconstruct the associated turbulent attractor and then follow its route towards chaos. We further show that the experimental attractor can be modeled by stochastic Duffing equations, that match the quantitative properties of the experimental flow, namely, the number of quasistationary states and transition rates among them, the effective dimensions, and the continuity of the first Lyapunov exponents. Such properties can be recovered neither using deterministic models nor using stochastic differential equations based on effective potentials obtained by inverting the probability distributions of the experimental global observables. Our findings open the way to low-dimensional modeling of systems featuring a large number of degrees of freedom and multiple quasistationary states.
\end{abstract}

DOI: 10.1103/PhysRevLett.119.014502

In the absence of external forcing, the only stationary state of a viscous flow is the trivial zero-velocity state. This state obviously respects all the symmetries of the system. Subject to a given forcing of intensity $\mu$, the trivial state can reach a nontrivial steady state (SS), the characteristics of which depend on $\mu$ and symmetry properties of the forcing. For $\mu$ below a critical value $\mu_{c}$, the SS is time independent and respects all the symmetries of the forcing compatible with the boundary conditions. As $\mu$ is increased past $\mu_{c}$, the SS gradually breaks all the forcing symmetries, resulting in fluid motion switching from time independent to periodic, chaotic, and ultimately reaching —at $\mu=\mu_{T} \gg \mu_{c}$-a turbulent state in which fluid motion is extremely irregular. This state, however, recovers all the symmetries of the forcing and the system in a statistical sense [1]. The turbulent flow is characterized by a dynamics with a large number of degrees of freedom, resulting from the wide range between the length scale at which energy is injected and the scale at which it is dissipated. This motivated Landau [2] to describe it as a quasiperiodic state, i.e., the superposition of a growing number of modes with incommensurate oscillation frequencies, resulting from an infinite number of bifurcations with increasing $\mu$. This picture was challenged by Ruelle and Takens [3], who proved that turbulent states are, in general, not quasiperiodic and conjectured that they could be described by a small number of degrees of freedom, i.e., by a low-dimensional "strange attractor" [3] on which all turbulent motions concentrate in a suitable phase space. This conjecture was fueled by seminal studies on prototype flows such a Taylor-Couette [4] or Rayleigh-Bénard convection [5,6], where it was shown that the transition to turbulence actually follows the traditional roads to deterministic chaos via the appearance of two or three characteristic frequencies and either quasiperiodicity with frequency locking, period doubling, or intermittency [7]. However, it was soon realized that this paradigm survives only during the transition to turbulence, namely, for $\mu_{c}<\mu \ll \mu_{T}$. All attempts [8-10] to find the strange attractor of a turbulence state failed. Does this mean that we must abandon all hope to apply tools from dynamical systems theory to turbulent flow?

We provide experimental evidence that the answer is negative using a laboratory model experiment in highly turbulent conditions. The key idea is that, even if a turbulent flow is characterized by a large number of degrees of freedom, some of them are less important than others and can be lumped into a noise term with a few relevant parameters. This motivates the shift towards the notion of random dynamical systems $[11,12]$ and stochastic chaos [13]. We are left with the problem of the identification of the relevant variables which 
represent the main properties of the steady state, in a statistical sense. Since the bifurcation in this system is connected with symmetry breaking, it is natural to choose this parameter so that it gives information about the symmetries of the turbulent steady state, in analogy with the usual order parameters in statistical physics. In our experiment, the order parameter is a global quantity measuring the response of the flow to an asymmetry of the flux of angular momentum. As the asymmetry is varied, the turbulent state becomes unsteady, and the formerly stable random attractor becomes unstable, in a sequence reminiscent of the topology changes of the Duffing attractor [14] with varying forcing amplitude.

We use the experimental setup as described in Ref. [15]. Turbulence is generated in a vertical cylinder of length $H=$ $180 \mathrm{~mm}$ and radius $R=100 \mathrm{~mm}$ filled with water and stirred by two coaxial, counterrotating impellers providing energy and momentum flux at the upper and lower ends of the cylinder. The impellers are made of disks of radius $0.925 R$, fitted with eight curved blades. It is thus not equivalent to drive the impellers in the scooping or unscooping direction. In the first case, previous experiments at fixed impeller frequencies evidenced the existence of two quasistationary states, with different symmetries [16]. Such multistability does not exist in the unscooping case. In the present Letter, we focus on the case where the impellers are driven in the scooping direction by two independent motors, operating in conditions such that the torques $C_{1}$ and $C_{2}$ applied by the flow onto the top and bottom impellers are constant. This procedure guarantees a stationary flux of angular momentum at each impeller. To quantify the global response of the flow to this forcing, we measure independently the rotating frequency $f_{1}$ and $f_{2}$ of the two impellers. With a typical mean applied torque $C=\left(C_{1}+C_{2}\right) / 2=1.68 \mathrm{~N} m$, we measure typical mean frequencies $f=\left(f_{1}+f_{2}\right) / 2$ of the order of $4<f_{s 1}<4.5 \mathrm{~Hz}$ or $6.5<f_{s 2}<7.5 \mathrm{~Hz}$ depending on the mean flow geometry. Our experiment being thermalized at a temperature $T=21 \pm 0.1{ }^{\circ} \mathrm{C}$, this corresponds to a typical Reynolds number $\mathrm{Re}=$ $2 \pi f R^{2} / \nu=3 \times 10^{5}$, far from the estimated critical Reynolds number for turbulence onset [17]: $\operatorname{Re}_{T}=3500$.

In what follows, we consider only data obtained for fixed values of the torques $C_{i}$. This means that there is only one free parameter characterizing the forcing. Because of the symmetry of our experimental setup, statistical-mechanical arguments [18] suggest the choice $\gamma(t)=\left[C_{1}(t)-C_{2}(t)\right] /$ $\left[C_{1}(t)+C_{2}(t)\right]$ as the control parameter. From now on, the parameter $\gamma$, corresponding to the $\mu$ parameter in our model, is understood as a time-averaged value of $\gamma(t)$ as $\gamma(t)$ experiences fluctuations in response to the turbulent flow. The amplitude of the fluctuations-measured as the standard deviation of $\gamma(t)$-is substantially independent of $\gamma$ [19]. We will see that the stochastic behavior of $\gamma(t)$ is the key to the concept of random attractors. When $\gamma=0$, the top and the bottom impeller are exchangeable, and we have checked that the turbulent state statistically follows this symmetry. As a result, the top and bottom rotating frequencies are statistically equal: The variable $\theta(t)=$ $\left[f_{1}(t)-f_{2}(t)\right] /\left[f_{1}(t)+f_{2}(t)\right]$ fluctuates around zero and characterizes the symmetries of the turbulent flow $[15,23]$.

The time series and the power spectral density (PSD) of the variable $\theta(t)$ for six different values of the parameter $\gamma$ are plotted in Fig. 1 (see also [19] for an enlargement of the spectral peaks). At $\gamma \approx 0$, the time series has the signature of decorrelated white noise, as evidenced by the flat spectrum. At larger values of $f$, the turbulent spectrum is superposed with characteristic frequencies $f_{s 2} \simeq 7 \mathrm{~Hz}$, the average impeller rotation frequency when the mean flow topology is quasisymmetrical (weakly symmetry broken). A frequency of the order of $2 f_{s 2}$ is also recognizable in the spectrum which then saturates to white noise for frequencies larger than $f_{N}=20 \mathrm{~Hz}$, where $N$ stands for noise. For other values of $\gamma$, the behavior is identical, with a shift of $f$ towards smaller and smaller values and the appearance of another frequency $f_{s 1}$, of the order of $4 \mathrm{~Hz}$, corresponding to the typical frequency of a new quasistationary state, with complete symmetry-broken mean flow topology (see the insets in Fig. 2). We first eliminate the irrelevant small-scale degrees of freedom at $f \geq f_{N}$ by performing a moving average of the time series over a time window of $30 \mathrm{~Hz}$. The corresponding time series is then analyzed using the local
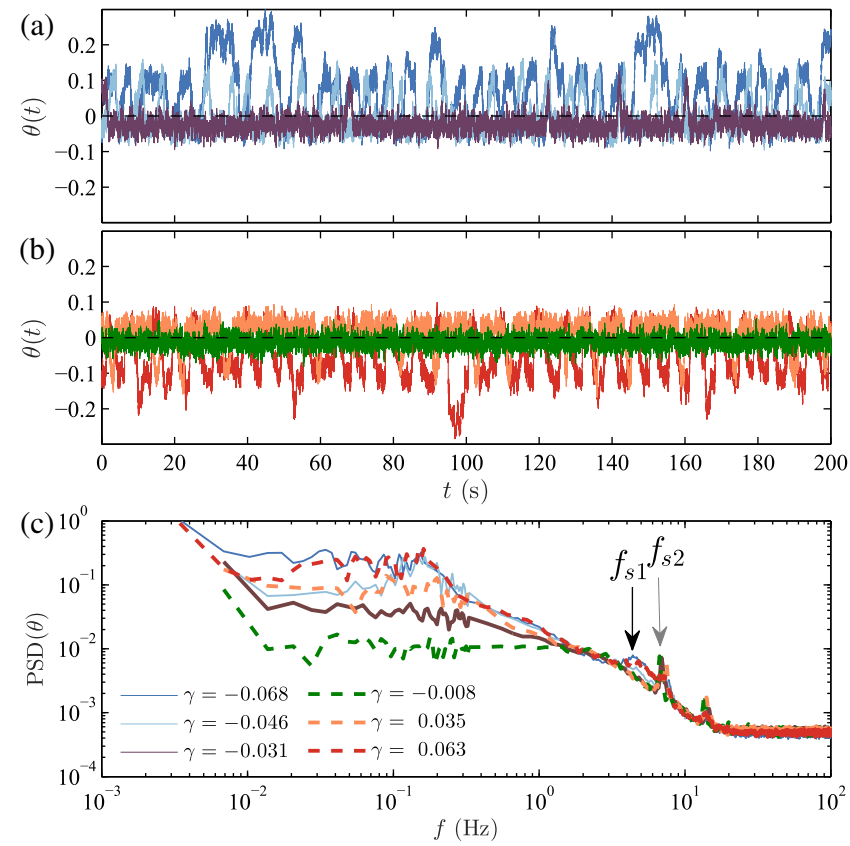

FIG. 1. (a),(b) Time series of the global observable $\theta$ reflecting the symmetries of the von Kármán turbulent flow experiment with torque forcing at $\operatorname{Re}=3 \times 10^{5}$. The dynamics of $\theta(t)$ for $\gamma \simeq 0$ consists of fluctuations around an average value. Instead, for higher values of $|\gamma|$, an irregular switching between different quasistationary states appears. (c) PSD of $\theta(t)$ for different values of $\gamma$. The black and gray arrows highlight the two spectral peaks $f_{s 1} \simeq 4.5 \mathrm{~Hz}$ and $f_{s 2} \simeq 7 \mathrm{~Hz}$ corresponding to the typical mean propeller frequency of the two quasistationary states. 


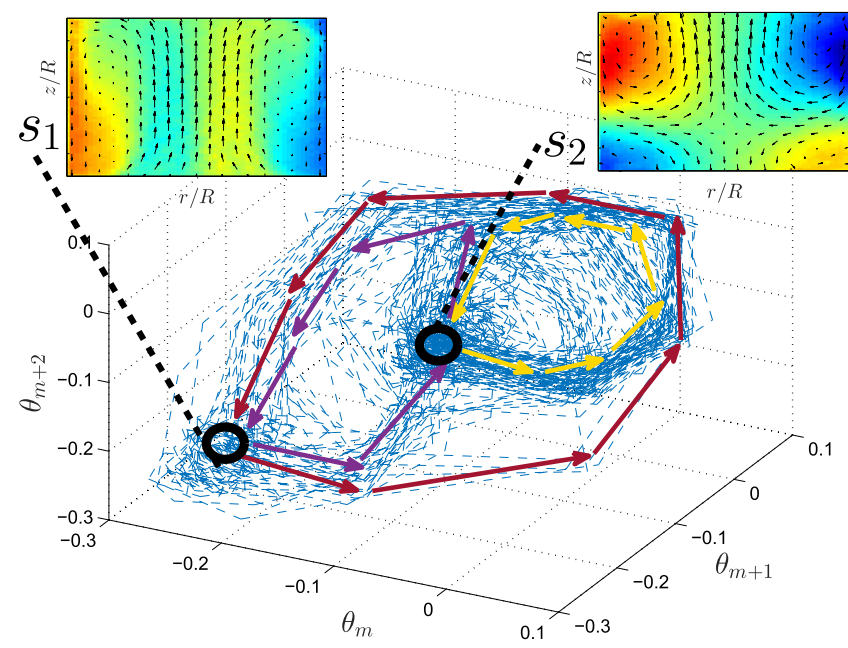

FIG. 2. The von Kármán turbulent flow attractor reconstructed for the experiment performed at $\gamma=0.067$. The attractor is obtained by embedding the peaks $\theta_{m}$ extracted from the time series $\theta(t)$ in a three-dimensional space. One can identify two quasistationary states (labeled as $s_{1}$ and $s_{2}$ ) corresponding to the average velocity fields (dark red insets, arrows, and color scale show, respectively, the in-plane and the orthogonal average velocity and three cycles (highlighted by colored arrows). The direction of the arrows indicates the only possible paths to switch from one state to the other. See also Supplemental video [19].

peak embedding procedure [24], by the extraction of the maxima $\theta_{m}$ (or minima, since the results do not change significantly) under the condition that subsequent maxima cannot fall within $10 \mathrm{~Hz}$ (see Fig. 6 in Ref. [19] for the portraits reconstructed with the delay methods [25]). Once the series of partial maxima is obtained, the attractor is visualized by plotting in an $n$-dimensional phase space, $\theta_{m}$, $\theta_{m+1}, \ldots, \theta_{m+n}$. The value of $n$, known as the embedding dimension, plays a crucial role in the applications of the dynamical systems theory to real data [26].

The embedding with $n=3$ for the experimental data with $\gamma=0.067$ is represented in Fig. 2 (see Supplemental video [19] for an animation of the dynamics). This attractor features two quasistationary states $s_{1}$ and $s_{2}$. The transitions from one state to another always follow one of the three cycles highlighted by the arrows, which indicate the only possible ways the system can switch.

The dynamics of $\theta$ shows a rich bifurcation diagram when $\gamma$ is varied. Some examples are shown in Figs. 3(a)-3(e). For $|\gamma| \sim 0$, the attractor is a random point attractor [Fig. 3(c)]. This is the analog of the trivial zero-velocity state of unforced viscous flow. For $0.02<|\gamma|<0.04$, a noisy periodic motion first appears [Figs. 3(b) and 3(d)]. For $|\gamma|>0.06$, this attractor bifurcates into a noisy chaotic attractor [Figs. 3(a) and 3(e)]. We have further checked that, when the constant torque forcing is applied on propeller rotating in the unscooping direction, only one trivial attractor is found, corresponding to the random point attractor. This means that the route to chaos observed in the scooping direction is a feature of the turbulent flow itself and not due to the electronic control of the torque.

We now look for the minimal dynamical system model capable of representing the dynamics of the experiment. The dynamics of $\theta(t)$ can be mimicked by an autonomous oscillator at frequency $f_{0}$, while the dynamics of $\gamma(t)$ induced by the turbulent fluctuations is represented by a (a) $\gamma=-0.068$

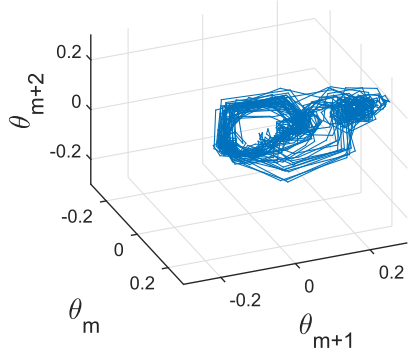

(f) $\quad \mu=-0.3$

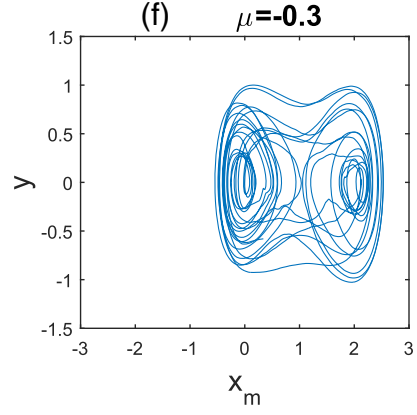

(b) $\gamma=-\mathbf{0 . 0 4 6}$

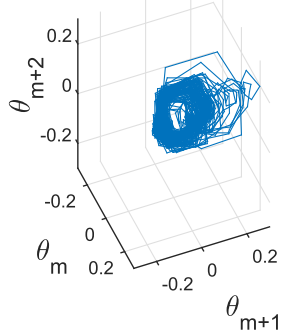

(g) $\mu=-\mathbf{0 . 1 5}$

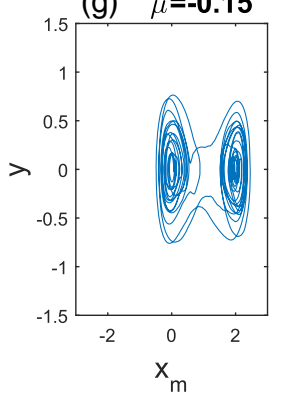

(c) $\gamma=-0.016$

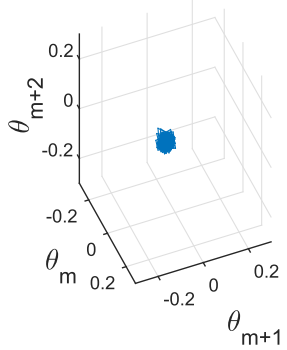

(d) $\gamma=0.056$
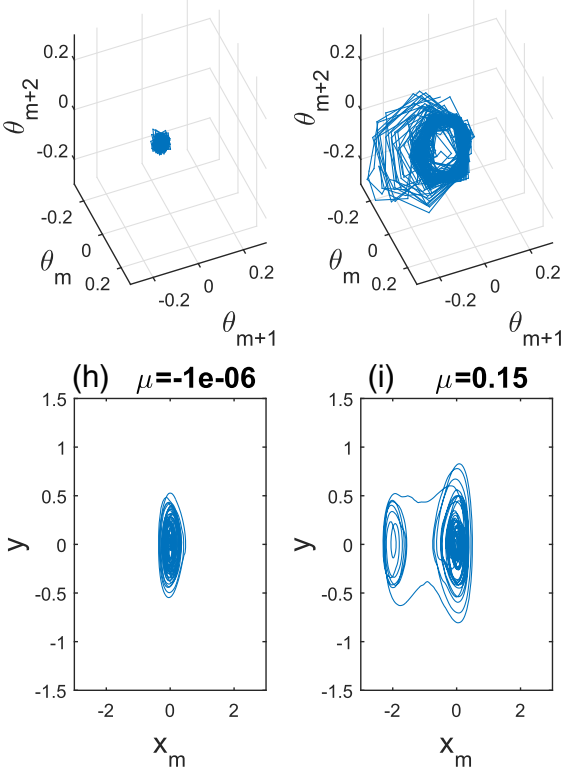

(e) $\gamma=0.064$
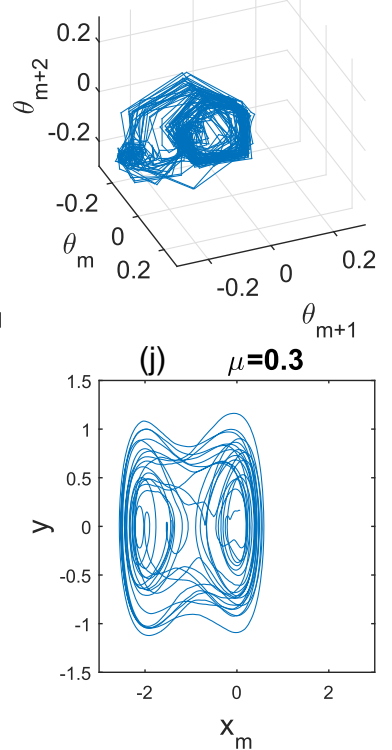

FIG. 3. (a)-(e) The von Kármán turbulent flow attractors for five different $\gamma$ values. From (c), the sequence shows how the attractor bifurcates symmetrically into a noisy periodic motion (b),(d) and into a noisy chaotic attractor (a),(e). (f)-(j) The same bifurcation sequence reproduced in the stochastic Duffing attractors for five different $\mu$ values. 
stochastic force. Moreover, the $\theta \rightarrow-\theta$ symmetry excludes the presence of a quadratic nonlinearity. The minimal model having such characteristics and capable of describing the sequence of bifurcations of the order parameter of the von Kármán flow $\theta(t)$ is the stochastic Duffing model, a nonautonomous dynamical system with two variables $x$ and $y=\dot{x}$ with random forcing $z$ obeying

$$
\begin{aligned}
& d x=y d t, \\
& d y=\left(-a y+x-x^{3}+z \sin \omega t\right) d t, \\
& d z=-\phi(z-\mu) d t+\sigma d W_{t},
\end{aligned}
$$

where $a=0.2, \phi=0.9, \sigma=0.2, \omega=1$, and $W_{t}$ is a Wiener process [27]. These parameters are taken in the chaotic regime of the Duffing equations to mimic the model dynamics. In our model, $x$ corresponds to the experimental $\theta$ and $\omega$ corresponds to $f_{0}$, while $z$ corresponds to the stochastic dynamics of $\gamma(t)$ modeled by the OrnsteinUhlenbeck process, the simplest stochastic model representing fluctuating dynamics of the control parameter: $\mu$ corresponds to $\gamma$, i.e., the time average of $\gamma(t), \sigma$ to the amplitude of the fluctuation of $\gamma(t)$, and $\phi^{-1}$ to the characteristic time needed by the system to restore the average $\gamma$ [19]. We integrate numerically the stochastic Duffing equations for different values of the control parameter $\mu$ with the code reported in Ref. [19]. Because of the symmetry $\theta \rightarrow-\theta$, we have two distinct Duffing attractors for the positive and negative values of $\gamma$. By observing that the quasistationary states of Eq. (1) are obtained for $x_{s}= \pm 1$, the two branches are recovered by shifting $x$ to $x_{m}=\operatorname{sgn}(\mu)(x-1)$. In Figs. 3(f)-3(j), we show the stochastic Duffing attractors in terms of $\left(x_{m}, y\right)$ for different values of $\mu$. As in the turbulent experimental system, there is a bifurcation from a random point attractor to random periodic attractors and to random strange attractors.

To check more quantitatively the analogy between the experimental system and the model, we have computed in both cases the effective dimension with the method proposed by Cao [28], comparing the experimental data $\theta(t)$ with a time series of $x(t)$ of the same length, for which we have repeated exactly the embedding procedure used for the von Kármán experiments. We obtain the effective dimension $n_{\text {eff }} \simeq 10$ from the experimental data and $n_{\text {eff }} \simeq$ 9 from the model. Moreover, the first Lyapunov exponent $\lambda_{1}$ from the data [Fig. 4(a)] and the first Lyapunov exponents computed from the model [Fig. 4(b)] show a qualitatively similar behavior as a function of the control parameters $\gamma$ and $\mu$. The stochastic behavior, induced by the fluctuation of the control parameter, is essential to get the full bifurcation diagram: By changing $\mu$ in the stochastic Duffing attractor, we observe smooth changes of the Lyapunov exponents, compatible with those observed in the experiments. In the deterministic case [19], the Lyapunov exponents exhibit discontinuous jumps for (a)

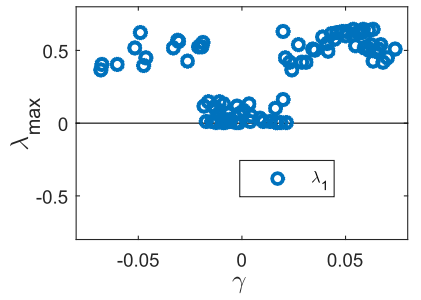

(b)

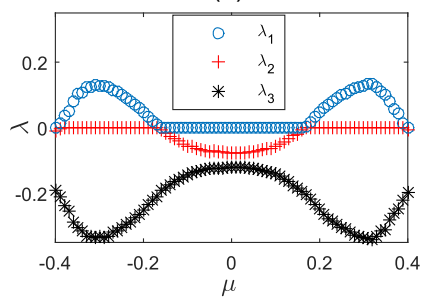

FIG. 4. (a) First Lyapunov exponents $\lambda_{1}$ measured for different $\gamma$ in the von Kármán turbulent flow (blue circles). For $\gamma \leq 0.02$, $\lambda_{1} \simeq 0$ corresponds to the experiments whose attractor is a random point attractor. For larger $|\gamma|, \lambda_{1}>0$ and noisy attractors are found. (b) First, second, and third Lyapunov exponents for different $\mu$ measured in the stochastic Duffing equation with $\sigma=0.2$. The behavior of $\lambda_{1}$ is qualitatively similar to that of the experiments. See also Figs. 13 and 14 in Ref. [19] for further robustness tests.

increasing $|\mu|$, so that the bifurcation diagram is incompatible with the one observed experimentally.

An essential feature of our reconstruction is the combination of tools from the classical dynamical system with ideas borrowed from stochastic modeling, where the influence of neglected degrees of freedom (here the small scales) are described through a noise. The resulting model is a dynamical system with a fluctuating control parameter. Such fluctuations strongly modify the bifurcation diagram of the original system, smoothing the variation of the Lyapunov exponents, in agreement with experimental findings [19]. As a result, the fluctuations of the order parameter, the transition rates, and the bifurcation structure respect the features experimentally observed. Therefore, the random dynamical systems framework is more suitable than the classical dynamical systems to describe our turbulent data. The noise by itself is, however, not a sufficient ingredient to reconstruct the full system dynamics. Inspection of the turbulent attractor in Fig. 2 may naively suggest that our system follows nothing else than a generalized Langevin model, described by the stochastic differential equations (SDEs), like in other turbulent systems $[29,30]$. In this approach, the effective potential may be found by inverting the probability distribution of the global observable (as measured, e.g., in Ref. [15]) to obtain the effective potential describing the fixed points. The transition between quasistationary states is then captured by the addition of a noise term, representing the interactions with smaller scales. This approach gives the stationary states with fluctuations but hardly returns the correct transition rates as shown by Ref. [31]. In fact, the implicit assumption that the potential is one-dimensional (e.g., by taking an overdamped limit) leaves only one possible transition path between quasistationary states. The turbulent attractor provided in Fig. 2 immediately suggests that this description is false: There is more than one path for the switch between $s_{1}$ and $s_{2}$, and the system dynamics 
cannot be reduced to a single SDE. So, while a SDE is superior to a classical deterministic model $[30,32]$, it fails to reproduce the exact dynamics in the phase space, as described by Lyapunov exponents and the transition rates. The random attractor model thus appears as the only candidate able to describe both statistical and dynamical features of our data.

We have provided experimental evidence that it is possible to describe the large-scale motion of a fully developed turbulent flow with a random dynamical system model with few degrees of freedom, if an appropriate observable reflecting the flow symmetry is selected. We claim that the large embedding dimensions which prevented the application of dynamical systems theory to turbulence arise from small-scale disturbances which can be modeled in terms of stochastic perturbations. This general picture reconciles the Landau [2] and RuelleTakens [3] descriptions of turbulence, the former being valid at small scales, and the latter describing the largescale motions. Our findings may be extended to other systems where chaos with large degrees of freedom plays a role, thereby defining the procedure to find attractors in geophysical fluid dynamics [8-10,33,34]. Like in our turbulent experiments, general oceanic or atmospheric circulations are characterized by general symmetry properties and small-scale dynamics that are possibly decorrelated. The main challenge is then to identify the relevant global observable that reflects the system symmetry and that can be used as an order parameter. Indeed, for some well-chosen atmospheric circulation index (see, e.g., [35]), the Duffing equation emerges as the minimal model for the description of midlatitude circulation dynamics.

The research leading to these results has been partially funded by the ERC Grant No. 338965-A2C2 and the Grantin-Aid for Scientific Research (C) No. 24540390, JSPS, Japan, and London Mathematical Laboratory External Fellowship, United Kingdom. We acknowledge N. Moloney for useful discussion and comments.

*Also at London Mathematical Laboratory, 14 Buckingham Street, London, WC2N 6DF, United Kingdom.

†rancois.daviaud@cea.fr

[1] U. Frisch, Turbulence: The legacy of A. N. Kolmogorov (Cambridge University Press, Cambridge, England, 1995).

[2] L. D. Landau, Dokl. Akad. Nauk SSSR 44, 339 (1944).

[3] D. Ruelle, and F. Takens, Commun. Math. Phys. 20, 167 (1971).

[4] A. Brandstater and H. L. Swinney, Phys. Rev. A 35, 2207 (1987).

[5] P. Bergé, Y. Pomeau, and C. Vidal, Order within Chaos (Wiley, New York, 1984).
[6] G. Ahlers, in Dynamics of Spatio-Temporal Cellular Structures (Springer, New York, 2006), pp. 67-94.

[7] P. Manneville, in Chaos-The Interplay between Stochastic and Deterministic Behaviour (Springer, New York, 1995), pp. 257-272.

[8] P. Grassberger, Nature (London) 323, 609 (1986).

[9] C. Nicolis and G. Nicolis, Nature (London) 311, 529 (1984).

[10] E. N. Lorenz, Nature (London) 353, 241 (1991).

[11] K. K. Lin and L.-S. Young, Nonlinearity 21, 899 (2008).

[12] M. D. Chekroun, E. Simonnet, and M. Ghil, Physica (Amsterdam) 240D, 1685 (2011).

[13] S. Lovejoy and D. Schertzer, Chaos Fract. Model. 96, 38 (1998).

[14] I. Kovacic and M.J. Brennan, The Duffing Equation: Nonlinear Oscillators and Their Behaviour (Wiley, New York, 2011).

[15] B. Saint-Michel, B. Dubrulle, L. Marié, F. Ravelet, and F. Daviaud, Phys. Rev. Lett. 111, 234502 (2013).

[16] F. Ravelet, L. Marié, A. Chiffaudel, and F. Daviaud, Phys. Rev. Lett. 93, 164501 (2004).

[17] F. Ravelet, A. Chiffaudel, and F. Daviaud, J. Fluid Mech. 601, 339 (2008).

[18] S. Thalabard, B. Saint-Michel, E. Herbert, F. Daviaud, and B. Dubrulle, New J. Phys. 17, 063006 (2015).

[19] See Supplemental Material at for http://link.aps.org/ supplemental/10.1103/PhysRevLett.119.014502, which includes additional figures and Refs. [20-23].

[20] E. N. Lorenz, J. Atmos. Sci. 20, 130 (1963).

[21] A. Wolf, J. B. Swift, H. L. Swinney, and J. A. Vastano, Physica (Amsterdam) 16D, 285 (1985).

[22] A. Wolf, https://fr.mathworks.com/matlabcentral/ fileexchange/48084-lyapunov-exponent-estimation-from-atime-series-documentation-added.

[23] B. Saint-Michel, F. Daviaud, and B. Dubrulle, New J. Phys. 16, 013055 (2014).

[24] N. H. Packard, J. P. Crutchfield, J. D. Farmer, and R. S. Shaw, Phys. Rev. Lett. 45, 712 (1980).

[25] F. Takens, in Dynamical Systems and Turbulence, Warwick 1980 (Springer, New York, 1981), pp. 366-381.

[26] H. Kantz and T. Schreiber, Nonlinear Time Series Analysis (Cambridge University Press, Cambridge, England, 2004), Vol. 7.

[27] K. Itô, Diffusion Processes (Wiley, New York, 1974).

[28] L. Cao, Physica (Amsterdam) 110D, 43 (1997).

[29] R. Benzi, A. Sutera, and A. Vulpiani, J. Phys. A 14, L453 (1981).

[30] E. Brown and G. Ahlers, Phys. Rev. Lett. 98, 134501 (2007).

[31] V. Lucarini, D. Faranda, and M. Willeit, Nonlinear Processes Geophys. 19, 9 (2012).

[32] E. Da Costa and R. Vautard, J. Atmos. Sci. 54, 1064 (1997).

[33] M. Göber, H. Herzel, and H. Graf, Ann. Geophys. 10, 729 (1992).

[34] P. Grassberger and I. Procaccia, Phys. Rev. Lett. 50, 346 (1983).

[35] M. H. Ambaum and L. Novak, Q. J. R. Meteorol. Soc. 140, 2680 (2014). 